\title{
Estimation of uncertainty in three dimensional coordinate measurement by comparison with calibrated points
}

\author{
J E Muelaner, Z Wang, O Martin, J Jamshidi and P G Maropoulos \\ Department of Mechanical Engineering, The University of Bath, Bath, UK \\ E-mail: J.E.Muelaner@bath.ac.uk
}

\begin{abstract}
This paper details a method of estimating the uncertainty of dimensional measurement for a three dimensional coordinate measurement machine. An experimental procedure was developed to compare three dimensional coordinate measurements with calibrated reference points. The reference standard used to calibrate these reference points was a fringe counting interferometer with a multilateration like technique employed to establish three dimensional coordinates. This is an extension of the established technique of comparing measured lengths with calibrated lengths. Specifically a distributed coordinate measurement device was tested which consisted of a network of Rotary-Laser Automatic Theodolites (R-LATs), this system is known commercially as indoor GPS (iGPS). The method was found to be practical and was used to estimate that the uncertainty of measurement for the basic iGPS system is approximately $+/-1 \mathrm{~mm}$ at a $95 \%$ confidence level throughout a measurement volume of approximately $10 \mathrm{~m} \times 10 \mathrm{~m} \times 1.5 \mathrm{~m}$.
\end{abstract}

Keywords: Coordinate Uncertainty, Coordinate Measuring Machine, CMM, iGPS, Indoor GPS, Spatial Analyzer, USMN

\section{Introduction}

Modern dimensional measurement systems do not simply measure lengths as is the case with traditional instruments such as micrometers and height gauges. Current industrial systems typically measure the three dimensional coordinates of points on objects and therefore verification by the measurement of lengths cannot ensure the traceability of all coordinate measurements made by the instrument [1].

The system verified in this work is a large volume frameless and distributed coordinate measurement machine that is made up of a network of RotaryLaser Automatic Theodolites (R-LATs), this system is known commercially as indoor GPS (iGPS). Each R-LAT consists of a transmitter and a sensor although many sensors may share a single transmitter. The transmitter utilizes a rotating head to sweep two fanned lasers through the measurement volume; the transmitter also houses a strobe which fires a timing signal covering 360 degrees of azimuth, as shown in Figure 1. The sensor is able to detect the incidence of these lasers and deduce the azimuth and elevation angle from the transmitter to the sensor from the time differences between the strobe and the two lasers reaching the sensor $[2,3]$.

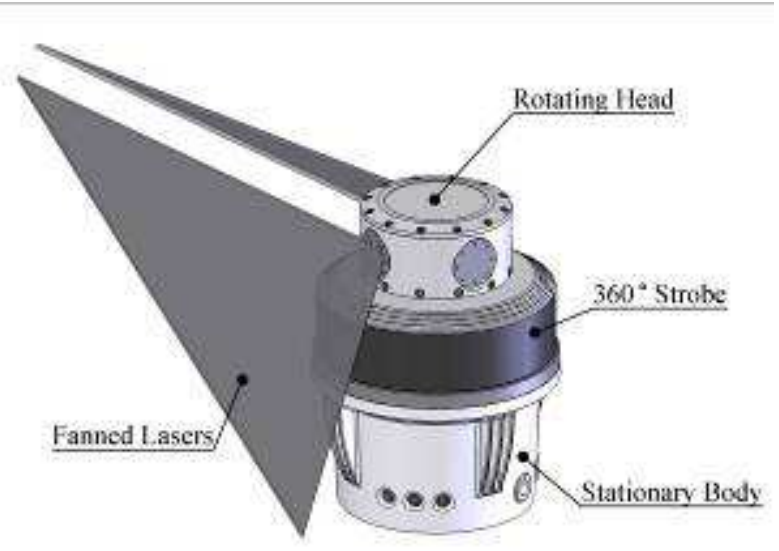

Figure 1: Main components of R-LAT transmitter 
In order to detect the three dimensional coordinates of the sensor it must receive optical signals from at least two transmitters. It is then possible to use triangulation to fix the position of the sensor assuming that the transmitter positions are known. Normally, a bundle adjustment [4] would be carried out as part of the setup procedure for the network. The bundle adjustment is used to establish the relative positions of the transmitters. If more than two transmitters are visible then some form of least squares fitting can be employed to reduce the uncertainty of the position.

Once the transmitter positions have been determined, the network of R-LATs then constitutes a large volume frameless coordinate measurement machine. This type of measurement network has advantages such as the ability of the one way communication, from the transmitter network to the sensors, to support a virtually unlimited number of sensors. Additionally, a sensor is able to move behind obstructions to the line of sight loosing and regaining connection to various transmitters without loosing connection to the network as a whole and not requiring any re-aiming of transmitters.

Typically, measurements are taken using a 'vector bar' shown in Figure 2. This is a calibrated device housing two sensors and with a 1.5" diameter steel sphere mounted at one end. The sensors and the sphere are mounted on a common axis. Since the position of the sensors can be calculated the position of the vector bar is also known and the rotation can also be calculated in two axes. The position of the sphere is therefore known enabling it to be used as a measurement probe.

\section{Verification strategy}

The body of literature concerning the verification of coordinate measurements is primarily concerned with comparison with calibrated lengths. The ISO 10360 standard for coordinate measuring machines [5] is a well established work applicable to conventional gantry based coordinate measurement machines (CMMs) using contact probing and operating in the discrete-point probing mode. There are currently two standards dealing with large volume frameless metrology instruments. The ASME standard for evaluating 'Laser-Based Spherical Coordinate Measurement Systems' [6] is applicable to Laser Trackers [7] and Laser Radars [8]. The German VDI/VDE 2634 Part 2 [9] is directly applicable to photogrammetric systems.

The above standards are based on a methodology of measuring calibrated lengths at various orientations in order to test the isolated and combined accuracy of the instruments' sub-systems. Such sub-systems are the probing error and $\mathrm{x}, \mathrm{y}, \mathrm{z}$ encoders on a $\mathrm{CMM}$, while on a laser tracker they are the two angle encoders, the interferometer and the probing error of the retro-reflector.

Applying the principle of isolating sub-systems previous work has been carried out to characterize the performance of an individual R-LAT [2]. The work reported here is concerned with the coordinate measurement performance of the complete iGPS system. This could be carried out using the established method of measuring calibrated lengths, however, since this would not ensure traceability of coordinate measurements made by the system it was decided to develop a method based on three dimensional coordinates.

The direct comparison of coordinates is not new, such an approach has, for example, been carried out to compare points measured on a surface with a laser scanner to points measured on the same surface with a conventional gantry type CMM [10]. However, such an approach does not give traceability since the coordinate measurements made by the CMM do not have direct traceability to a length standard.

A tracking interferometer has been used to measure the distance to a CMM head from multiple positions. These distances were then used to calculate coordinates using multilateration. Multilateration is a technique of combining multiple one-dimensional measurements to give three-dimensional measurements. It is therefore similar to the more widely known technique of triangulation but while triangulation combines multiple angular measurements, multilateration combines multiple length measurements. The difference between the nominal and the measured coordinates was then used to create an error map [11]. The work described here follows essentially the same method with a few notable exceptions; standard industrial instruments are used such as a laser tracker and the measurements are used for verification by an assessment of measurement uncertainty [12] rather than for error mapping.

The approach employed in this work involves the use of kinematic nests, shown in Figure 2, to allow the repeatable positioning of both the reference measurement system and the system undergoing verification. These nests are commonly used to position the spherically mounted retro-reflectors (SMRs) used by Laser Trackers. Although the use 
of such nests will introduce additional uncertainty, this can be shown to be relatively small and quantifiable through repeated measurement with the reference system.

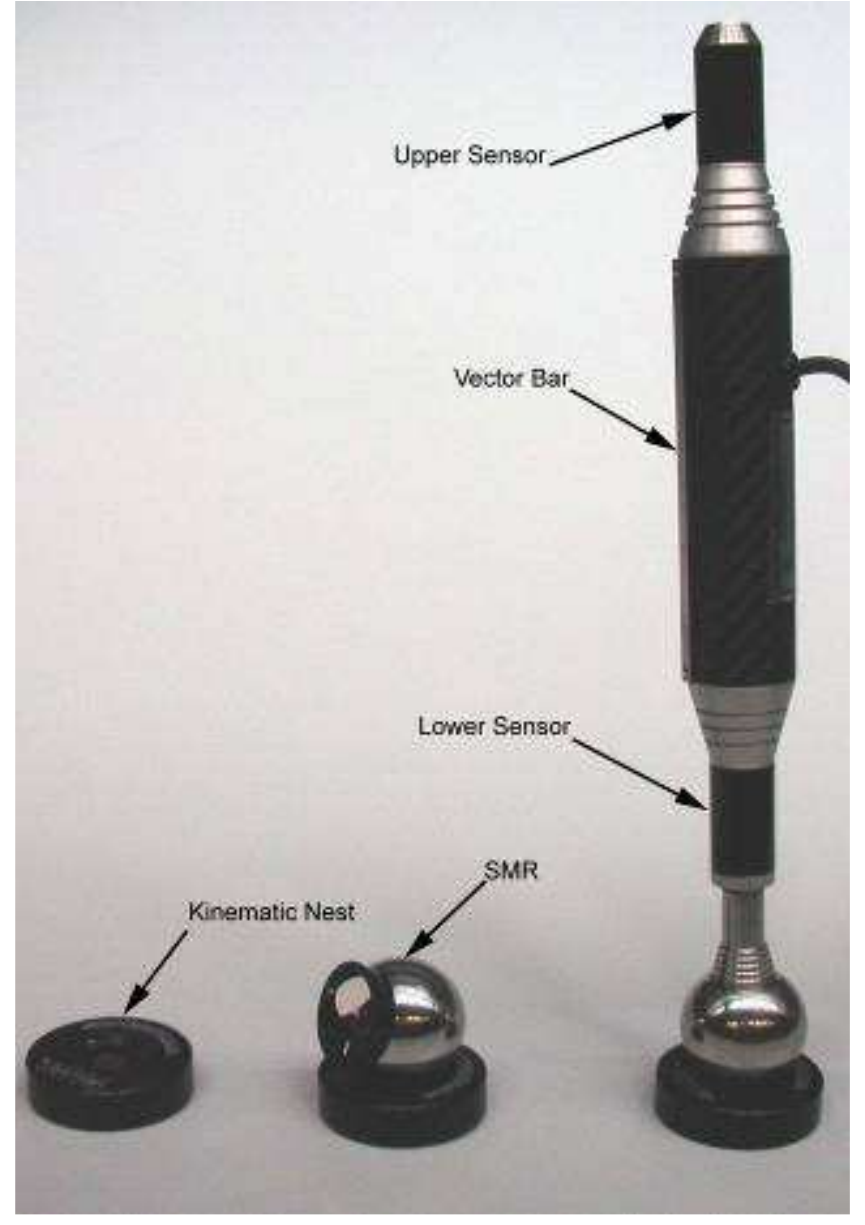

Figure 2 : Kinematic nests with SMR and vector bar

\section{Experimental Procedure}

Two tests were carried out at different locations both of which represented typical aerospace production environments. Although there were some differences between the tests the basic procedure was the same. The actual setup used for the tests carried out at the Bath LIMA [13] is shown in Figure 3.

The traceability route for this uncertainty evaluation is through the use of a calibrated laser tracker system with a technique similar to multilateration used to obtain the best, length measurement, performance from the laser tracker system.

\subsection{Instrumentation Used}

The R-LAT network used was the Metris iGPS system with the Workspace interface with a manufacturer specified measurement uncertainty of $0.2 \mathrm{~mm}$ at 2 sigma.

The laser tracker system used to create the reference network was the Faro Xi. This has a manufacturer specified standard measurement uncertainty of $10 \mu \mathrm{m}+0.4 \mu \mathrm{m} / \mathrm{m}$.

\subsection{R-LAT Network Setup}

The R-LAT network was setup using the supplied interface software [14] according to the user manual [15]. This involved positioning and starting the transmitters, setting various parameters and then connecting a vector bar to the network. The network consisted of 4 transmitters.

A bundle adjustment was carried out as specified in the user manual [15]. This involved taking measurements using the vector bar at 8 observation points within the working volume. The bundle was initially calculated using the known distance between the sensors on the vector bar to apply scale. Accurate lengths between two kinematic nests were then calibrated using an interferometer and the scale was reapplied by taking measurements of these nests with the vector bar.

\subsection{Coordinate Network Calibration}

The reference coordinates were created using kinematic nests designed to accept a 1.5 " steel ball. A number of nests were glued to the concrete slab forming the floor of the test venue while others were mounted on either a granite table or theodolite stands.

Following the initial layout the coordinates of each kinematic nest were measured using a Laser Tracker. Measurements were taken from a number of positions allowing the results to be combined to improve accuracy using a technique similar to multilateration which is explained in section 4 . The number of positions differed between the tests. 


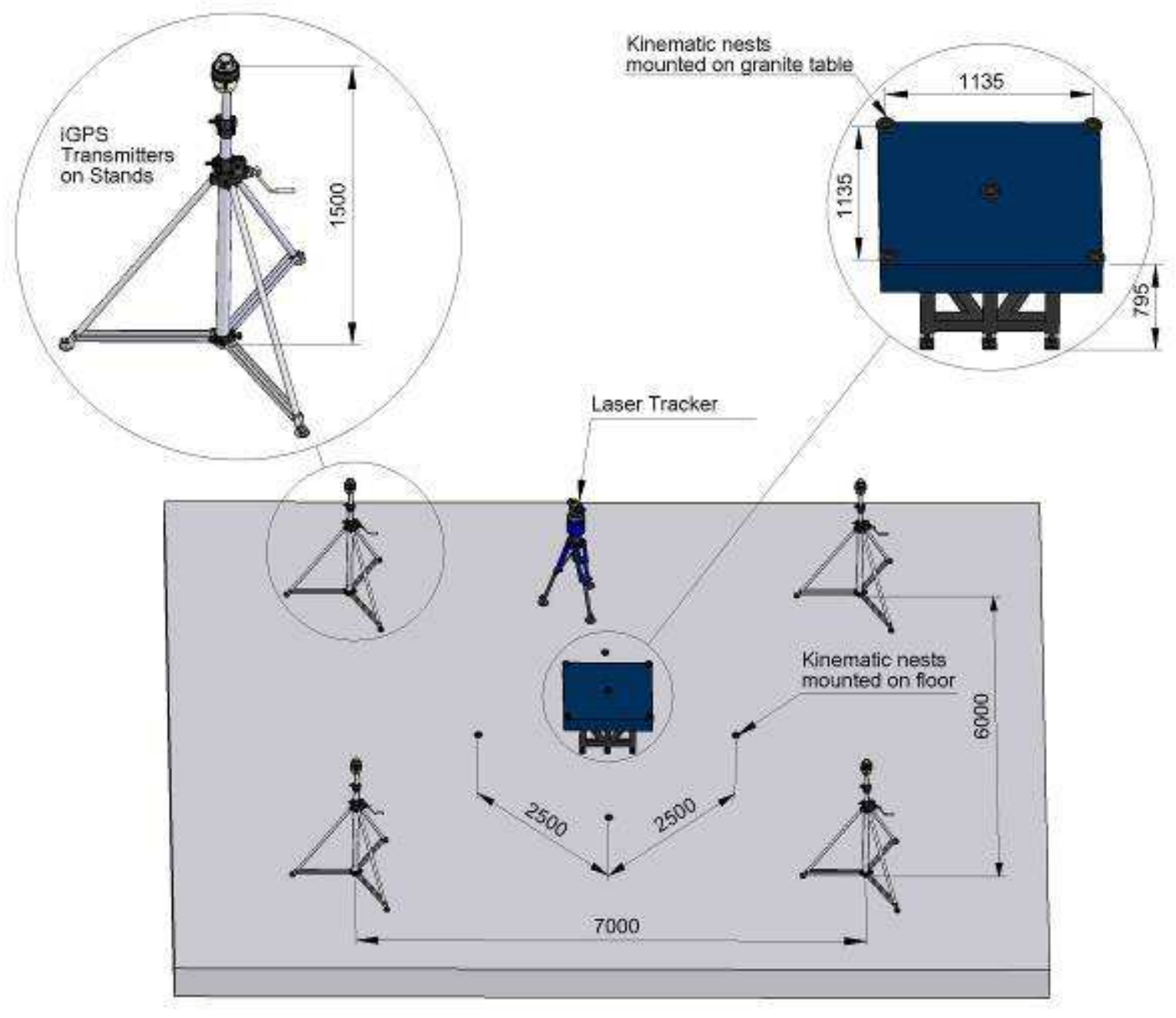

Figure 3: Test setup for tests at the Bath LIMA

\subsection{Replicated Measurements}

Following the coordinate network calibration the iGPS Vector Bar was used to make repeated measurements of the position of each kinematic nest. A 1.5" probe tip was used which was the same size as the SMR used for the Laser Tracker calibration. The points measured by the two methods are therefore equivalent. Each point was measured in turn using the Vector Bar and the measurements were then repeated a number of times measuring all the points in a circuit. The number of measurements of each point differed between the tests.

The system has a sampling frequency of approximately $40 \mathrm{~Hz}$ resulting from the rotational velocity of the transmitter heads. Due to the substantial effects of environmental disturbances such as turbulence on optical measurements [16] more accurate measurements can be made by averaging over a period of time. A single measurement of a coordinate position was therefore considered to be an average of 80 instantaneous measurements, this was regarded as giving a good compromise between accuracy and operation time [2].

An interface program was created to automate the measurement process and export of text files for further analysis. This interface software used rotation data from the Vector Bar to ensure that the Vector Bar was orientated vertically to within \pm 2 Degrees. The graphical user interface is shown in Figure 4. 


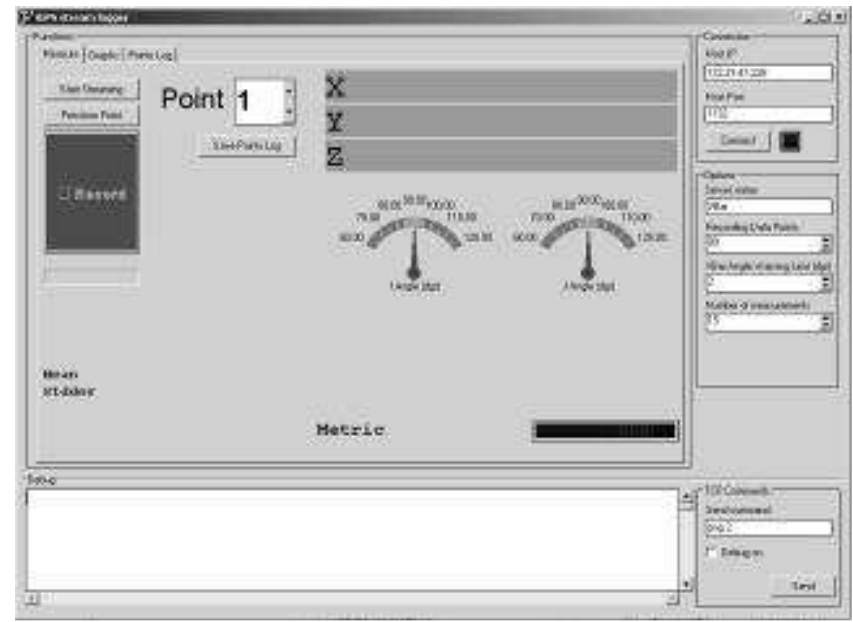

Figure 4 : Interface software used to collect measurement data

\subsection{Individual Experiments}

Although both tests used the experimental procedure detailed above there were some differences in the details of the setup and calibration. These differences are detailed in Table 1. The SMR nests for tests carried out at Bath's Laboratory for Integrated Metrology and Assembly (LIMA) were located on the floor and a granite table. For the tests carried out at a large aircraft assembly area at Airbus Broughton, the nests were located on the floor and on theodolite stands.

Table 1 : Details of individual experiments

\begin{tabular}{|c|c|c|}
\hline Location & Bath LIMA & $\begin{array}{c}\text { Airbus } \\
\text { Broughton }\end{array}$ \\
\hline Date & $27 / 2 / 08$ & $4 / 3 / 08$ \\
\hline Laser Tracker Positions & 2 & 5 \\
\hline No. of Points & 9 & 15 \\
\hline Measurements per Point & 25 & 6 \\
\hline Transmitter Layout & $9 \mathrm{~m} \mathrm{x} \mathrm{7} \mathrm{m}$ & $12 \mathrm{~m} \times 12 \mathrm{~m}$ \\
rectangle & square \\
\hline $\begin{array}{c}\text { Scale Lengths } \\
\text { Used to Bundle }\end{array}$ & $5.6 \mathrm{~m}$ & $\begin{array}{c}8.3 \mathrm{~m}, 8.9 \mathrm{~m} \\
9.4 \mathrm{~m}, 11.2 \mathrm{~m}\end{array}$ \\
\hline
\end{tabular}

\section{Analysis of Results}

The analysis of results consisted of two stages. Firstly measurements of the reference coordinate network were made from multiple Laser Tracker positions and combined using weighted best fitting to minimize the uncertainty for the point positions and a Monte Carlo simulation to estimate the uncertainty of this reference. The actual iGPS measurements were then analysed with respect to this reference to estimate the uncertainty of the iGPS system.

\subsection{Reference Coordinate Network}

The measurements from multiple Laser Tracker positions were combined into a single survey of the coordinate network using a technique which produces results similar to multilateration, reducing the coordinate uncertainty. This was achieved using a commercial code, Unified Spatial Metrology Network (USMN) which runs in the Spatial Analyzer (SA) software produced by New River Kinematics (NRK). This combines Monte Carlo simulation (MCS) with best fitting of point clouds $[17,18]$.

The fundamentals of this technique are that the uncertainty of a particular measurement is simulated using knowledge of the position of the measurement instrument and the non-isotropic uncertainty of the instrument. The simulation is of the Monte Carlo type with repeated simulated measurements made, each consisting of the nominal measurement value with random noise added to it.

Each series of measurements of all the points from a single measurement station represents one point group. All of the point groups can then be best fitted to each other using a least squares minimization algorithm. The best fitting is weighted giving less weight to coordinates with higher uncertainty. In this way points with, for example, a large standard deviation in the z-direction are allowed to deviate more in the $z$-direction from fitting to the corresponding points. The point groups are best fit to one another for each measurement in the uncertainty field in turn creating a new composite uncertainty field of the weighted best fits.

The repeated best fitting to generate a composite uncertainty field represents a second level of Monte Carlo simulation which is used to find the combined uncertainty for the coordinate measurements from multiple stations. Since the uncertainty of measurements taken using a Laser Tracker is known to be considerably better in range than in angle [19] the distance measurements will be given greater weight than the angle derived measurements. The end result of this approach is therefore similar to multilateration. It is not however pure multilateration since the angle derived measurements are still used to some extent.

The process employed by USMN is best illustrated using a simple 2-Dimensional example. In the example below 4 points are measured by 2 instruments. Each instrument measures two points in common with the other as well as a unique point as shown in Figure 5. 


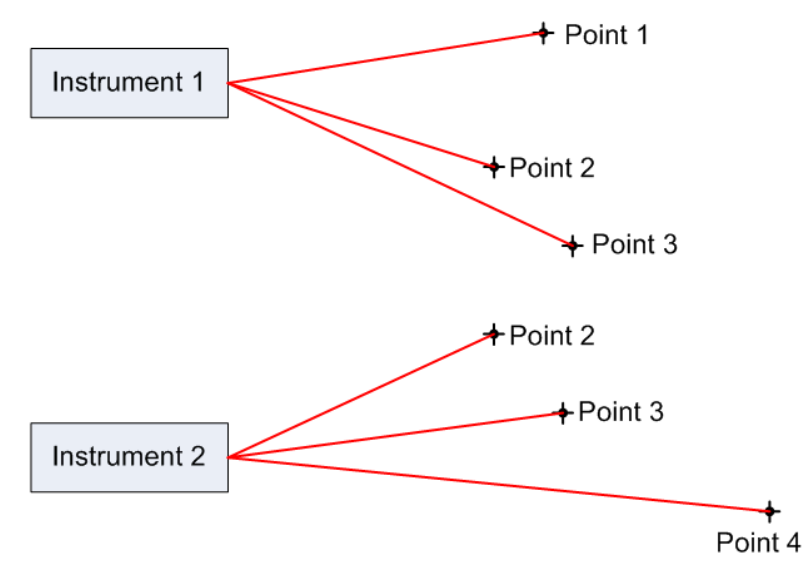

Figure 5 : Example of 2-dimensional measurement of 4 points using 2 instruments

Each measurement of a point is simulated using a Monte Carlo technique. In its simplest form this might involve adding a randomly generated, normally distributed error with standard deviation equal to the measurement uncertainty, to each coordinate of each nominal measurement to give simulated measurement coordinates. The actual simulation carried out by USMN is somewhat more complicated since the standard deviations used for each coordinate of each measurement is first calculated to represent the range dependent and anisotropic uncertainty of coordinate measurements. Repeated simulations of each measured point are calculated to create a point cloud around the nominal point - this is referred to as an Uncertainty Field and is illustrated in Figure 6. The Uncertainty Field is a graphical representation of the uncertainty of each measurement and additionally allows the uncertainty of the measurement to be estimated by directly measuring the dispersion of these points. It should be noted that the calculation of uncertainty from the Uncertainty Field at this stage would be circular and pointless but it will be shown that in subsequent steps of the process that it becomes a valuable technique.
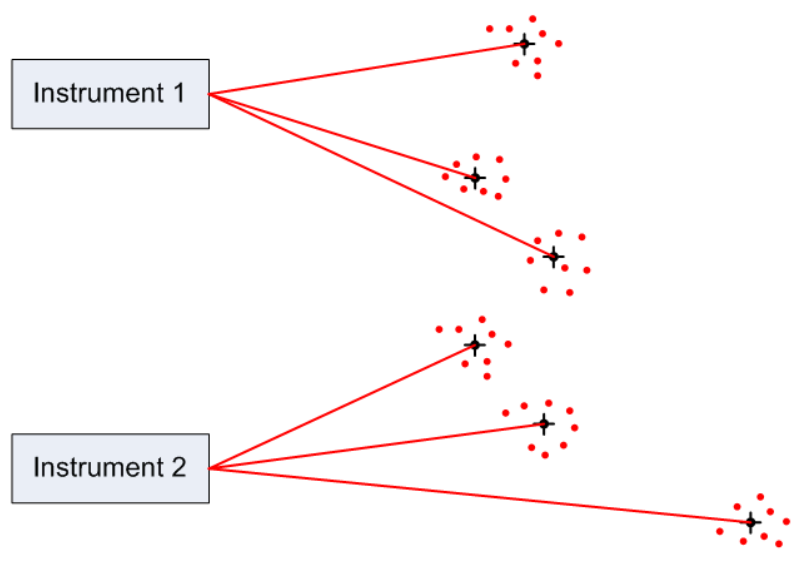

Figure 6 : Measured points with simulated 'uncertainty fields' around them
With the instruments in their individual coordinate systems the location of point 4 relative to point 1 is not known. By best fitting the common (nominal) points using a least squares minimization algorithm instrument 2 can be located relative to instrument 1 . All measured points are transformed with the instrument which measured them. This locates point 4 at the correct distance from point 1 and instrument 2 at the correct position relative to instrument 1 as shown in Figure 7.

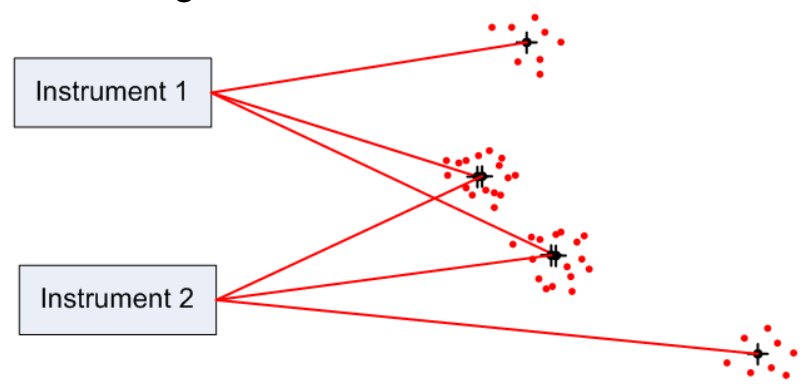

Figure 7 : Instrument 2 located relative to instrument 1 by best fitting the common points

At this stage the model gives information about the location of points within a network and not simply with respect to one instrument. It also gives a representation of the uncertainty of each measured point with respect to the instrument which measured it. What the model does not give at this stage is an accurate representation of the uncertainty of point 4 with respect to point 1 . The reason for this is that the best fitting used to locate instrument 2 was based purely on the nominal point and did not consider the uncertainty of the points.

If each of the simulated measurements in the uncertainty fields is numbered so that the uncertainty field for point 2 as measured from instrument 1 would be made up of points 1-2-1, 1-2-2 etc and the uncertainty field for point 2 as measured from instrument 2 would be made up of points 2-2-1, 2-2-2 etc as shown in Figure 8. The number convention is therefore Instrument-Point-Simulated Measurement. Instrument 2 can be located relative to instrument 1 by best fitting 1-2-1 to 2-2-1 and 1-3-1 to 2-3-1 to give a simulated position for instrument 2 and associated measurements. 


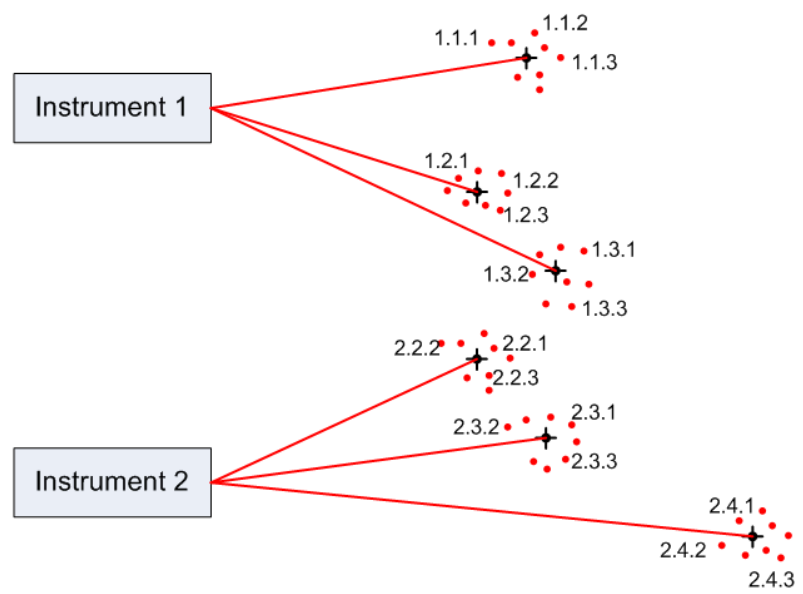

Figure 8 : Simulated 'Uncertainty Fields' with the first three simulations of each point labelled using the convention Instrument-Point-Simulated Measurement

This process can be repeated to locate instrument 2 relative to instrument 1 by best fitting $1-2-2$ to $2-2-2$ and $1-3-2$ to $2-3-2$ to give another simulated position for instrument 2 and associated measurements. This process can be repeated to build-up an uncertainty field for the network as a whole, known as the USMN Composite Group as shown in Figure 9.

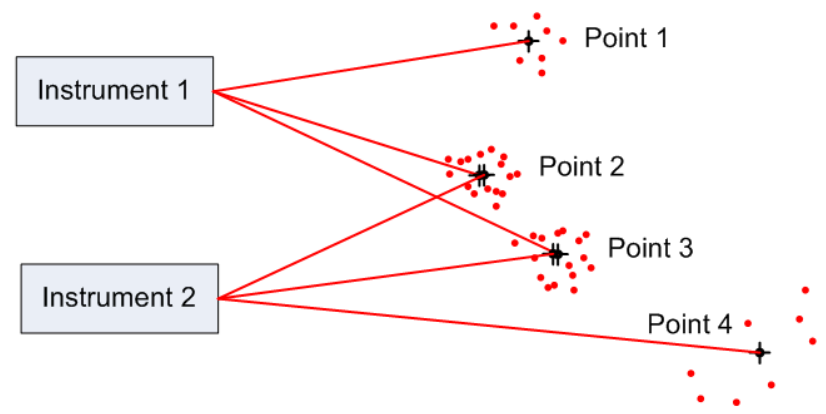

Figure 9 : USMN composite group showing increased uncertainty at point 4 due to 'chaining' of instruments

Each simulated measurement from instrument 1 and each simulated and transformed measurement from instrument 2 is added to the USMN composite group. Finally the USMN composite simulation group is used to find the mean value for each point and the standard deviation. It is now possible to evaluate the uncertainty of point 4 relative to point 1 .

There is a further complexity which was omitted up to this point in order to simplify the explanation. It was already mentioned above that the coordinate uncertainties are range dependent and anisotropic. The uncertainty of each coordinate is therefore used to weight the best fitting of point clouds so that the greater the uncertainty the less effect that particular coordinate will have on the transformation applied to the whole point cloud. The complete USMN process is summarized in Figure 10. Simulations were run with 500 replications.

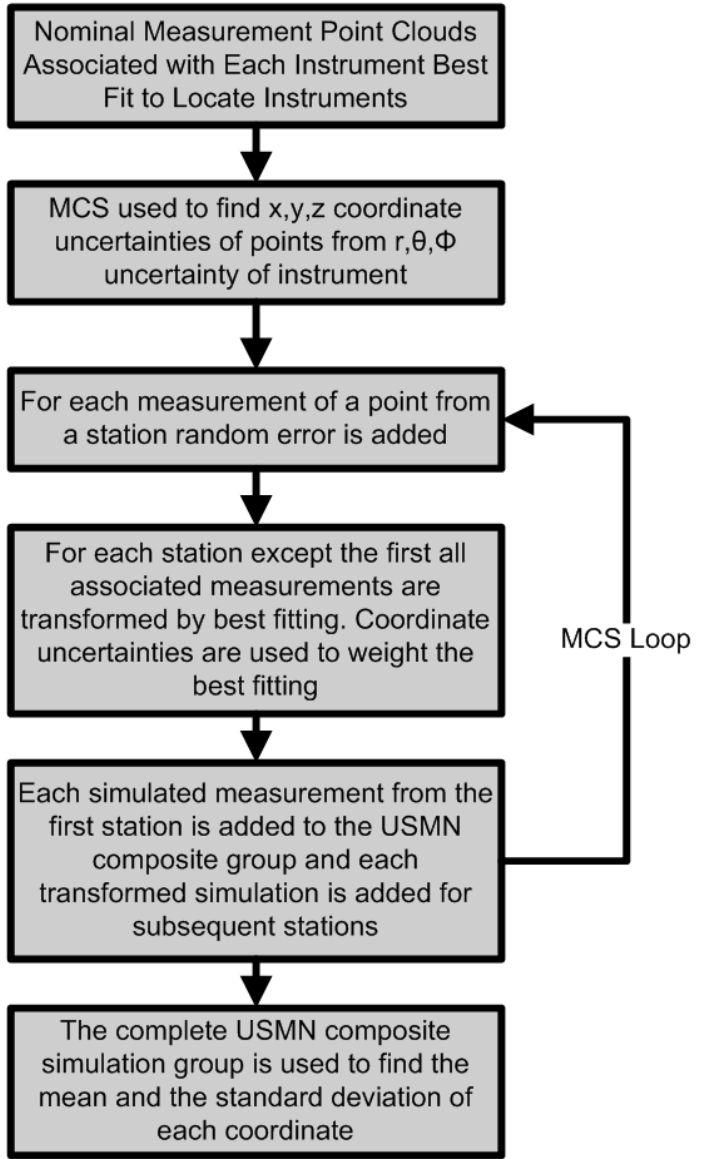

Figure 10 : Summary of the USMN process

\subsection{Analysis of IGPS Measurements}

An uncertainty budget for the iGPS system is shown in Table 2. The reference standard uncertainty includes components of uncertainty relating to the Laser Tracker system used, the drift nest repeatability and the SMR centring tolerances. It was calculated using USMN as explained above. The functional relationship between these quantities is of the form.

$Y=X_{1}+X_{2} \ldots X_{N}$

Therefore the components of uncertainty related to each quantity can be simply added in quadrature with sensitivity coefficients of 1 for all components.

The uncorrected systematic errors appear to vary randomly throughout the measurement volume and were therefore be modelled as normal distributions rather than the rectangular distribution which would more typically be used for systematic errors.

The uncertainty budget includes components of uncertainty due to environmental effects such as the expansion of the concrete slab due to a variation in temperature of approximately 1 degree over a $10 \mathrm{~m}$ 
length, and refractive effects due to temperature

metre. gradients in the air of approximately 1 degree per

Table 2 : Uncertainty budget for iGPS system as tested at Broughton

\begin{tabular}{|c|c|c|c|c|}
\hline Component & $\begin{array}{l}\text { Value } \\
(\mu \mathrm{m})\end{array}$ & Distribution & $\begin{array}{c}\text { Standard } \\
\text { Uncertainty } \\
(\mu \mathrm{m})\end{array}$ & Contribution \\
\hline Reference Standard & 25 & Normal & 25 & $0.1 \%$ \\
\hline Repeatability of iGPS (RSS StDev) & 377 & Normal & 377 & $51 \%$ \\
\hline Rounding errors of iGPS & 0.5 & Rectangular & 0.4 & $0.00 \%$ \\
\hline Expansion of Concrete slab & 120 & Rectangular & 84.9 & $1 \%$ \\
\hline Refractive effects & 10 & Rectangular & 7.1 & $0.01 \%$ \\
\hline Uncorrected Systematic Error & 318 & Normal & 318 & $29 \%$ \\
\hline \multicolumn{3}{|c|}{ Combined Standard Uncertainty } & 503 & \\
\hline \multicolumn{3}{|c|}{ Expanded Uncertainty at $95 \%$ Confidence Level } & 1,006 & \\
\hline
\end{tabular}

The mean of the replicated measurements of each point was calculated and these averaged measurements were best fitted to the calibrated positions using a least squares minimization algorithm. The distance between the mean position as measured using the iGPS network and the calibrated point position after best fitting all the points was then calculated, this uncorrected systematic error appeared to vary randomly throughout the measurement volume.

Table 2 shows not only the uncertainty budget but also the contribution of each component to the combined uncertainty. This clearly shows that the majority of components have a negligible effect with only the repeatability of the iGPS system and the uncorrected systematic error making a significant contribution.

The length between each point position was also calculated and a comparison made in this way between the Laser Tracker Calibration and the iGPS measurements. The uncertainty budget was calculated in the same way for the length based measurements in the same way as for the coordinate measurements.

\section{Results}

The results presented here illustrate a direct comparison of coordinate measurements (Table 3) with a length based verification strategy (Figure 11 \& Figure 12).

Table 3 : Coordinate uncertainty for tests carried out

\begin{tabular}{|c|c|c|}
\hline $\begin{array}{c}\text { Component - Standard } \\
\text { Uncertainties }\end{array}$ & Bath LIMA & Broughton \\
\hline Reference Standard $(\mu \mathrm{m})$ & 25 & 25 \\
\hline Repeatability of iGPS $(\mu \mathrm{m})$ & 289 & 377 \\
\hline $\begin{array}{l}\text { Rounding errors of iGPS } \\
\qquad(\mu \mathrm{m})\end{array}$ & 0.4 & 0.4 \\
\hline $\begin{array}{l}\text { Expansion of Concrete } \\
\qquad \text { slab }(\mu \mathrm{m})\end{array}$ & 84.9 & 84.9 \\
\hline Refractive effects $(\mu \mathrm{m})$ & 7.1 & 7.1 \\
\hline Systematic Error $(\mu \mathrm{m})$ & 505 & 318 \\
\hline $\begin{array}{l}\text { Combined Standard } \\
\text { Uncertainty }(\mu \mathrm{m})\end{array}$ & 590 & 503 \\
\hline $\begin{array}{l}\text { Expanded Uncertainty at } \\
\qquad 95 \%(\mu \mathrm{m})\end{array}$ & 1,180 & 1,006 \\
\hline
\end{tabular}




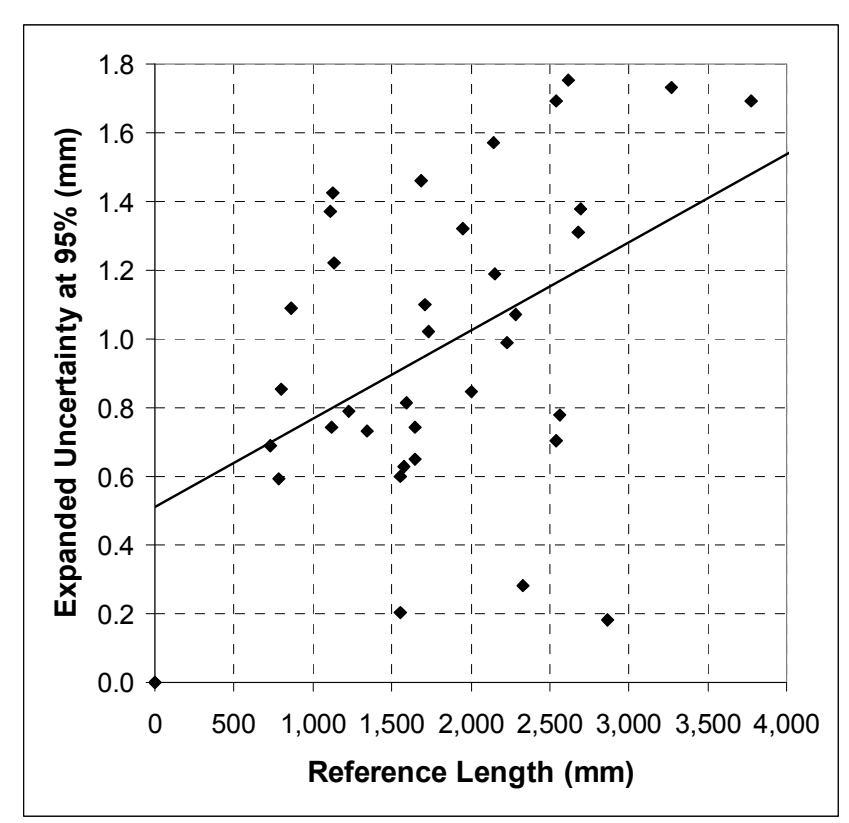

Figure 11 : Comparison of lengths for tests carried out at Bath LIMA

The performance of the system was somewhat different on the two tests. The Bath LIMA tests showed a somewhat higher uncertainty and very little evidence for the uncertainty being dependent on the reference length as can be seen in Figure 11. The tests carried out at Airbus Broughton showed the system to have a generally lower expanded uncertainty. There was also somewhat more evidence of a length dependence as shown in Figure 12.

The difference in performance between tests may be partially explained by differences in the setup procedure. The differences were most pronounced when the uncertainty was calculated based on the repeatability of each point individually. The setup of the iGPS system at Airbus Broughton involved a larger number of lengths to scale the bundle adjustment. However, this would be expected to affect the system bias (systematic error) rather than the variability. It is also possible that the small number of replicates (just six per point) gave a standard deviation that was not representative of the true variability of the system. If the repeatability is calculated as the standard deviation of all of the points together then the results become more consistent with the results of the tests conducted in the Bath LIMA.

The expanded uncertainties calculated using length based verification were similar to the coordinate results discussed above. The length based results showed a considerably wider range of results as shown in Figure 11 and Figure 12.

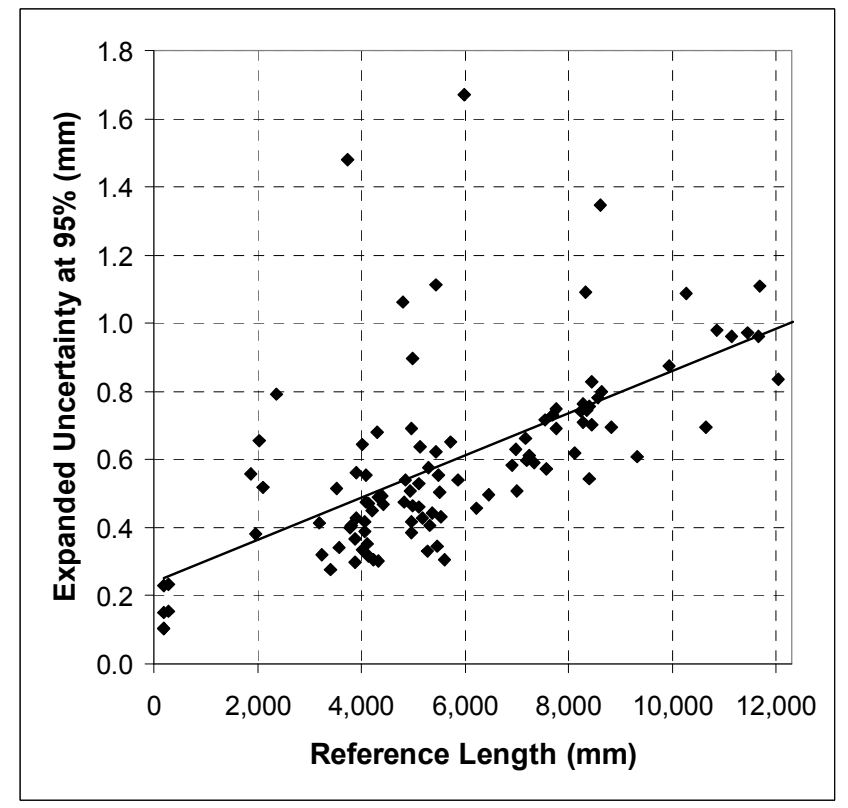

Figure 12: Comparison of lengths for tests carried out at Airbus Broughton

The test results indicate an expanded coordinate uncertainty magnitude at a $95 \%$ confidence level of between $+/-0.8 \mathrm{~mm}$ and $+/-1.1 \mathrm{~mm}$. It should be noted that these tests were carried out using a basic version of the iGPS interface software which is not the state of the art interface.

Since a weak relationship between reference length and uncertainty was seen and the systematic effects appeared to vary randomly throughout the measurement volume these figures apply throughout the measurement volume of approximately $10 \mathrm{~m} \mathrm{x} 10$ $\mathrm{m}$ by $1.5 \mathrm{~m}$. The uncertainty interval given represents results seen in different tests.

\section{Conclusions}

Previous work to verify the performance of an R-LAT showed that the angular uncertainty of an individual transmitter receiver pair was approximately 0.5 arc seconds at a 95\% confidence level [2]. From basic trigonometry this is equivalent to 0.012 to $0.048 \mathrm{~mm}$ within the $5-20 \mathrm{~m}$ range. This is considerably less than the total uncertainty of the network acting as a coordinate measuring machine is shown be this work. These results indicate that there are additional sources of uncertainty inherent in the combined system. This shows the importance of combined system tests in addition to isolated tests of sub-systems.

The technique demonstrated here is appropriate for the verification of all types of coordinate measurement instrument. The calibration of points for these tests was carried out using a Laser Tracker. If pure multilateration was applied so that only the 
interferometric measurements were used in the calibration of the reference points then traceability would be improved. Future work will develop a more rigorous mathematical approach in order to ensure traceability of the point calibration.

\section{Acknowledgements}

The authors wish to thank E B Hughes and A B Forbes of the National Physical Laboratory for advice and support given, $\mathrm{Mr}$ Mike O'Sullivan and $\mathrm{Mr}$ Geraint Jones of Airbus UK for hosting the tests at Broughton and staff from Metris for their technical inputs.

This research has been carried out as part of the IdMRC at the Department of Mechanical Engineering of the University of Bath, under EPSRC Grants No. $\mathrm{EP} / \mathrm{E} 002617 / 1$ and EP/E00184X/1. The participation of NPL was funded under a Joint Industry Project and the financial support of DIUS is acknowledged.

\section{References}

1 Flack, D. Measurement Good Practice Guide No. 42: CMM Verification. (National Physical Laboratory, 2001).

2 Muelaner, J.E., Wang, Z., Jamshidi, J., Maropoulos, P.G., Mileham, A.R., Hughes, E.B. and Forbes, A.B. Uncertainty of Angle Measurement for a Rotary-Laser Automatic Theodolite (R-LAT). IMechE, Part B: J. Engineering Manufacture, 2008, 223(B3), 217-229.

3 Hedges, T.M., Takagi, H., Pratt, T. and Sobel, M.J. Position Measurement System and Method Using Cone Math Calibration. In Patent, U.S., ed (Arc Second, 2003).

4 Triggs, B., Mclauchlan, P., Hartley, R. and Fitzgibbon, A. Bundle Adjustment - A Modern Synthesis. Vision Algorithms: Theory and Practice, International Workshop on Vision AlgorithmsCorfu, Greece, 1999).

5 BSI. Geometrical Product Specifications (GPS) - Acceptance and reverification tests for coordinate measuring machines (CMM) - Part 2: CMMs used for measuring size. BS EN ISO 1036022002).

6 ASME. Performance Evaluation of LaserBased Spherical Coordinate Measurement Systems. B89.4.192006).

7 Lau, K., Hocken, R. and Haight, W. Automatic Laser Tracking Interferometer System for
Robot Metrology. pp. 100-102Interlaken, Switz, 1985).

8 RICHARD, S. and KENDALL, B. Chirped coherent laser radar system and method. 2006).

9 VDI/VDE-Gesellschaft Mess- und Automatisierungstechnik. Optical 3D measuring systems - Imaging systems with point-by-point probing. VDI/VDE 2634 Part 22002).

10 Anchini, R., Di Leo, G., Liguori, C., Paolillo, A., Pietrosanto, A. and Strazzullo, G. Metrological verification of 3D scanners: A preliminary approach. p. 649108 (SPIE -International Society for Optical Engineering, Bellingham WA, WA 98227-0010, United States, San Jose, CA, United States, 2007).

11 Schwenke, H., Franke, M., Hannaford, J. and Kunzmann, H. Error mapping of CMMs and machine tools by a single tracking interferometer. CIRP Annals - Manufacturing Technology, 2005, 54(1), 475-478.

12 BSI. General Metrology - Part 3: Guide to the expression of uncertainty in measurement (GUM). PD 6461-31995).

13 Bath, T.U.o. The Lab for Integrated Metrology Applications. Bath, UK, 2009).

14 Metris.2007. WorkSpace. v 6.0.114.2

15 Metris. Training document iGPS \& WorkSpace. 2007).

16 Estler, W.T., Edmundson, K.L., Peggs, G.N. and Parker, D.H. Large-scale metrology - An update. CIRP Annals - Manufacturing Technology, 2002, 51(2), 587-609.

17 New River Kinematics.2007. SpatialAnalyzer. V

18 Calkins, J.M. Quantifying Coordinate Uncertainty Fields in Coupled Spatial Measurement Systems Mechanical Engineering, p. 226 (Virginia Polytechnic Institute and State University, Blacksburg, 2002).

19 Faro. Faro Laser Tracker Xi. 2007). 